\title{
Proposição de procedimento de detecção sistemática de perturbações oftalmológicas em escolares*
}

\section{A proposal for a procedure for the detection of ophthalmic disturbances in school children}

\author{
Rosely Moralez de Figueiredo**, Eloisa Cristina dos Santos***, Ivonete Ap. Almas de Jesus ${ }^{* * * *}$, \\ Rosa Maria Castilho**** , Edmar Vieira dos Santos"*****
}

\begin{abstract}
FIGUEIREDO, R. M. de et al. Proposição de procedimento de detecção sistemática de perturbações oftalmológicas em escolares. Rev. Saúde Pública, 27: 204-9, 1993. Proposição de um procedimento de detecção sistemática de perturbações oftalmológicas em escolares, com participação do pessoal da escola. A partir de levantamento realizado em escolas estaduais que oferecem ciclo básico, da cidade de São Carlos, SP (Brasil), foi proposta a realização de teste de acuidade visual. Nove escolas participaram do estudo. Os professores das escolas foram treinados e realizaram os testes de acuidade visual e estrabismo nos seus alunos, de forma padronizada. Das 2.025 crianças testadas, $88,1 \%$ apresentaram níveis de acuidade visual maiores que 0,8 e uma prevalência de estrabismo de $2,17 \%$. Finalizada a aplicação dos testes, cada escola apresentou propostas para aplicação sistemática do teste. As propostas têm em comum a realização dos testes pelos professores com a supervisão pelos coordenadores do ciclo básico, cabendo à enfermeira assessoria a todos os níveis de atividades do programa.
\end{abstract}

Descritores: Testes visuais, utilização. Triagem de massa, normas. Saúde escolar. Distúrbios da visão, prevenção.

\section{Introdução}

As consequêencias da deficiência visual poderiam ser atenuadas ou mesmo evitadas se fossem detectadas a tempo, ou seja, durante o período de desenvolvimento do aparelho visual, que se encer$\mathrm{ra}$, segundo Vaughan e Asbury ${ }^{17}$, aos 7 anos, e Gasperin e col. ${ }^{5}$, aos 6 anos.

\footnotetext{
* Subvencionado pelo CNPq (Conselho Nacional de Desenvolvimento Científico e Tecnológico) - Processo $\mathrm{n}^{9}$ 80154/373GL. Apresentado nos Congressos Brasileiros Unificados de Saúde Escolar, São Paulo, 1989

** Pós-Graduanda da Faculdade de Ciências Médicas da Universidade Estadual de Campinas (UNTCAMP) . Campinas, SP - Brasil.

*** Prefeitura Municipal de Campinas, Campinas, SP Brasil

**** Santa Casa de Misericórdia de São Carlos - São Carlos, SP - Brasil

***** Serviço Nacional de Aprendizagem Comercial - São Carlos, SP - Brasil

****** Departamento de Enfermagem da Universidade Federal de São Carlos - São Carlos, SP - Brasil
}

Separatas/Reprints: R. M. de Figueiredo - Rua Mogi Mirim, 250 Ap. 34 - Jd. Campos Elíseos - 13051080 - Campinas, SP - Brasil

Edição subvencionada pela FAPESP. Processo Medicina 93/ 0208-5.
A necessidade de deteç̧ão precoce é citada por Temporini ${ }^{14}$, José e Temporini ${ }^{6}$, José e col. ${ }^{7}$ e Sperandio ${ }^{12}$, que aconselham que a medida de acuidade visual (AV) seja feita no grupo populacional dos escolares mais jovens, ou seja, da préescola ou ensino elementar. Em muitos países desenvolvidos, segundo Temporini ${ }^{14}$, o teste de AV é obrigatório em torno de 3-4 anos, sendo realizado por oftalmologista, ortoptista, pediatra, enfermeiro ou outro profissional devidamente treinado. Esse citado autor refere que "em informe da OMS a acuidade visual é considerada o indicador mais pronto da função visual, sendo que a aplicação desse teste năo requer treinamento prolongado dos examinadores e nem uso de equipamentos sofistificados".

Sabe-se que toda criança deveria ser submetida a exame oftalmológico ainda em idade pré-escolar, onde o poder de resolução dos problemas detectados seria muito maior, uma vez que o aparelho visual da criança ainda está em pleno desenvolvimento ${ }^{6}$. Entretanto, Temporini ${ }^{16}$ reconhece que a escola é uma instituição aglutinadora de grande número de crianças e que a aplicação do teste nessa fase permite uma cobertura parcial da lacuna existente entre nascimento e o ingresso na escola.

Considerando a amplitude e conseqüências do problema apontado pelos vários autores, constatou-se que em 5 das 19 escolas estaduais de pri- 
meiro grau da cidade de São Carlos, SP, o teste de AV não era realizado de forma sistemática ou mesmo esporádica.

Com base nessa observação foi realizado o presente trabalho, que teve como objetivo propor um procedimento de deteç̧ão sistemática de perturbaçőes oftalmológicas em escolares, com a participação do pessoal da escola.

\section{Metodologia}

O trabalho foi feito em duas fases: na primeira foram aplicados os testes de AV e estrabismo e, na segunda, foram levantadas propostas para implantação de programa de identificação sistemática de problemas oftalmológicos.

Verificou-se que eram 12 escolas que ofereciam a primeira e segunda séries do ciclo básico, sendo que nove delas participaram da primeira fase do presente trabalho.

Foram utilizados um roteiro* onde foram registradas as regras para execução dos testes de $\mathrm{AV}$ e estrabismo ${ }^{11}$ e um formulário* onde foram registrados os resultados dos testes de todos os alunos de cada escola, além da tabela de Snellen.

O contato com cada escola foi feito através de uma visita ao seu diretor, em que foram expostos os objetivos do projeto, discutindo-se a melhor forma de execução para escola em questão. Após o projeto ser aceito pelo diretor, este o encaminhava para o responsável pela coordenação do ciclo básico, que reunia todos os professores para apresentação do projeto e discussão sobre a melhor forma de sua execução na escola. Todos os professores eram treinados para aplicação dos testes de AV e estrabismo.

Em relação ao preparo da criança, foi solicitado à escola que se fizesse primeiramente um esclarecimento sobre o teste na sala-de-aula com todos os alunos. Posteriormente, ao aplicar o teste em cada criança, esses esclarecimentos deveriam ser repetidos caso surgissem dúvidas.

Após o treinamento, o roteiro era deixado na escola para consulta posterior e o formulário era distribuído para cada professor.

Os professores aplicaram os testes nos seus alunos, utilizando os horários das aulas de Educação Física e Educação Artística, uma vez que essas disciplinas contam com professores específicos, e os professores do ciclo básico permanecem na escola durante o mesmo horário. O local escolhido para a realização dos testes foi o pátio.

O roteiro e formulário utilizados poderão ser obtidos diretamente com oprimeiro autor do presente artigo.
Foram mantidos contatos permanentes com a escola e uma vez terminada a aplicação dos testes, os formulários preenchidos eram devolvidos. Com base no mesmo, eram selecionadas as crianças que apresentavam AV inferior a $0,8 \mathrm{em}$ um ou ambos os olhos, para que fossem submetidas à repetição do teste. Se confirmada a redução da AV, pela repetição do teste, os pais ou responsáveis eram convocados e informados sobre o resultado do teste e da necessidade de consulta a um serviço especializado. Os serviços oftalmológicos existentes na cidade eram informados aos pais, ficando a critério dos mesmos a escolha ao qual recorrer.

Na segunda fase do trabalho foram feitos contatos com professores, coordenadores do ciclo básico e diretores de 12 escolas, em reuniōes semanais ou encontros individuais, para obtenção de sugestões para implantação do programa de identificação sistemática de problemas oftalmológicos. No contato com as duas primeiras escolas foi feita a pergunta sobre qual seria a melhor forma de garantir que os testes de AV e estrabismo fossem aplicados todos os anos na escola. Nos contatos seguintes, as propostas obtidas daquelas escolas eram apresentadas aos professores, coordenadores ou diretores para que os mesmos pudessem analisá-las, apoiar as sugestões apontadas ou sugerir novas propostas. Das 12 escolas com as quais foi feito contato, 11 participaram dessa segunda fase.

A análise estatística dos dados seguiu Beiguel$\operatorname{man}^{1}$.

\section{Resultados}

Na primeira fase do trabalho participaram 94 professores que realizaram os testes em 2.025 crianças.

A Tabela 1 apresenta a frequiência de resultados de crianças testadas para $\mathrm{AV}$, que está representada por uma fração na qual o numerador é a distância do teste $(5 \mathrm{~m})$ e o denominador a distância em que 0 optotipo apresentando forma, no ponto nodal do olho, o ângulo de $1 \mathrm{~min}$. A AV igual a 5/5 ou 1,0 foi considerada para todas as crianças que apresentaram esse "grau" (terminologia popular para designar o valor dióptrico de uma lente) em ambos os olhos. Nas demais drioptrias, definida como unidade de vergência ou unidade óptica com a qual se exprime o poder focal de uma lente e que se define como o inverso (em metros) de uma das distâncias focais de tal lente, foi considerado o menor "grau" apresentado por um dos olhos.

Verificou-se (Tabela 1) que com exceção da AV igual a 0,6, a frequiência de crianças cai à medida que se reduz a acuidade visual. A diferença 
Tabela 1. Freqüéncia de resultados do teste de acuidade visual em crianças que frequ̇entam ciclo básico em nove escolas estaduais de São Carlos, SP. (Primeira lase)

\begin{tabular}{crc}
\hline Acuidado visual & $N^{2}$ de crianças & $\begin{array}{c}\text { Frequéncia relativa } \\
(\%)\end{array}$ \\
\hline 1,0 & 1.300 & 64,20 \\
0,9 & 266 & 13,14 \\
0,8 & 218 & 10,76 \\
0,7 & 91 & 4,49 \\
0,6 & 45 & 2,22 \\
0,5 & 47 & 2,32 \\
0,4 & 18 & 0,89 \\
0,3 & 18 & 0,89 \\
0,2 & 14 & 0,69 \\
0,15 & 5 & 0,25 \\
0,1 & 3 & 0,15 \\
\hline Total & 2.025 & 100,0 \\
\hline
\end{tabular}

maior está entre as AV iguais a 1,0 e 0,9 ; entre este e a AV igual a 0,8 e entre este e os demais.

A Tabela 2 apresenta a frequiência de resultados de crianças que foram submetidas ao teste de AV dos olhos direito e esquerdo, segundo sexo. Com exceção da AV igual a 0,3 no olho direito das crianças do sexo masculino e AV igual a 0,3 e 0,5 no olho direito das do sexo feminino, houve diminuição progressiva da freqüência de ambos os sexos à medida que aumenta a deficiência visual em ambos os olhos.

Verificou-se que não existem diferenças significativas entre os valores de $A V$, tanto para o olho direito como para o esquerdo em ambos os sexos (teste de heterogeneidade: $x_{(10)}^{2}=3,087, P>0,5$ para o olho direito; $x_{(10)}^{2}=-3,005, P>0,01$ para $O$ olho esquerdo).

Tabela 2. Freqüência de resultados do teste de acuidade visual (AV), para os olhos direito $\theta$ esquerdo, em crianças do ciclo básico de nove escolas estaduais de São Carlos. (Primeira lase)

\begin{tabular}{|c|c|c|c|c|c|c|c|c|}
\hline \multirow{3}{*}{$\begin{array}{l}\text { AV } \\
\text { Sexo }\end{array}$} & \multicolumn{4}{|c|}{ Olho direito } & \multicolumn{4}{|c|}{ Olho esquerdo } \\
\hline & \multicolumn{2}{|c|}{ Masculino } & \multicolumn{2}{|c|}{ Feminino } & \multicolumn{2}{|c|}{ Masculino } & \multicolumn{2}{|c|}{ Feminino } \\
\hline & $N^{2}$ & $\%$ & $\mathrm{~N}^{2}$ & $\%$ & $N^{2}$ & $\%$ & $\mathrm{~N}^{2}$ & $\%$ \\
\hline $\begin{array}{l}1,0 \\
0,9 \\
0,8 \\
0,7 \\
0,6 \\
0,5 \\
0,4 \\
0,3 \\
0,2 \\
0,15 \\
0,1\end{array}$ & $\begin{array}{r}755 \\
102 \\
112 \\
39 \\
21 \\
17 \\
5 \\
9 \\
2 \\
1 \\
1\end{array}$ & $\begin{array}{r}70,96 \\
9,59 \\
10,53 \\
3,67 \\
1,98 \\
1,60 \\
0,46 \\
0,84 \\
0,19 \\
0,09 \\
0,09\end{array}$ & $\begin{array}{r}674 \\
121 \\
84 \\
31 \\
9 \\
15 \\
9 \\
10 \\
6 \\
1 \\
1\end{array}$ & $\begin{array}{r}70,14 \\
12,60 \\
8,75 \\
3,23 \\
0,93 \\
1,56 \\
0,93 \\
1,04 \\
0,62 \\
0,10 \\
0,10\end{array}$ & $\begin{array}{r}753 \\
115 \\
106 \\
38 \\
22 \\
16 \\
4 \\
4 \\
2 \\
2 \\
2\end{array}$ & $\begin{array}{l}70,78 \\
10,80 \\
9,96 \\
3,58 \\
2,07 \\
1,50 \\
0,37 \\
0,37 \\
0,19 \\
0,19 \\
0,19\end{array}$ & $\begin{array}{r}668 \\
119 \\
77 \\
43 \\
17 \\
17 \\
8 \\
5 \\
4 \\
2 \\
1\end{array}$ & $\begin{array}{r}69,53 \\
12,39 \\
8,02 \\
4,48 \\
1,76 \\
1,76 \\
0,83 \\
0,52 \\
0,41 \\
0,20 \\
0,10\end{array}$ \\
\hline
\end{tabular}

Total $1.064 \quad 100 \quad 961 \quad 100 \quad 1.064 \quad 100 \quad 961 \quad 100$
O estrabismo foi encontrado em $2,17 \%$ das 2.025 crianças testadas. Não foi possível estabelecer relaçōes entre os valores de acuidade visual e estrabismo devido os registros não terem sido sistematizados por parte dos professores que aplicaram os testes.

$\mathrm{Na}$ segunda fase participaram 26 professores, 6 coordenadores do ciclo básico e 5 diretores das 11 escolas. Foram apresentadas 3 propostas e respectivas justificativas, representadas pelas letras A, B e C (Tabela 3). A proposta A foi apresentada por 15 professores, 2 coordenadores e 1 diretor de 3 escolas. O item $a$ enfatiza as atividades do coordenador do ciclo básico na implantação do programa de AV. O item $b$ faz referência à época da realização do teste de AV na escola, mencionando o início do ano letivo como o mais indicado para a realização do mesmo.

A proposta B e sua justificativa foi apresentada por 11 professores, 4 coordenadores do ciclo básico e 3 diretores de 7 escolas. Além dos itens $a$ e $b$ da proposta A, a proposta B inclui o item $c$ que faz referência à assessoria da enfermeira aos coordenadores do ciclo básico na realização das atividades de planejamento, treinamento e acompanhamento da aplicação dos testes de $\mathrm{AV}$ pelos professores.

A proposta $\mathrm{C}$ e respectiva justificativa foi apresentada pelo diretor de 1 escola. A diferença desta proposta em relação às anteriores reside na enfermeira prestar assessoria apenas ao coordenador geral do cíclo básico, ao invés dos coordenadores de cada escola.

\section{Discussáo}

Os resultados encontrados pelo presente estudo em relaçăo à AV igual a 1,0 são bastante superiores aos encontrados por Vilar e col. ${ }^{18}$ ao relatarem que de um total de 5.809 crianças de escolas públicas do Estado do Ceará, o percentual encontrado foi de $38,9 \%$, em média. Qual seria a razão de tanta diferença entre esses dados? A hipótese de falta de coordenação motora apontada pelos autores, pode ser válida no caso das crianças da faixa etária entre 6 a 8 anos, enquanto que a melhora espontânea pode ter ocorrido com as crianças da faixa etária entre 12 e 14 anos. Entretanto, essas hipóteses não conseguem explicar a diferença nas AV encontradas em crianças dos Estados do Ceará e São Paulo.

A prevalência de problemas oftalmológicos encontrada em outros trabalhos ${ }^{5,18}$ é igual ou superior ao encontrado no presente estudo, que foi de $11,9 \%$. 
Tabela 3. Propostas e respectivas justificativas apresentadas pelas pessoas envolvidas na Segunda fase do trabalho, mediante a pergunta sobre qual seria a melhor maneira de garantir a aplicação dos testes de AV e estrabismo, todos os anos, na escola.

\begin{tabular}{|c|c|c|c|}
\hline & A & B & $C$ \\
\hline $\begin{array}{l}P \\
R \\
0 \\
P \\
0 \\
S \\
T \\
A \\
S\end{array}$ & $\begin{array}{l}\text { a) Os coordenadores do ciclo } \\
\text { básico ficam responsáveis por: } \\
\text { - inclusão do programa de AV } \\
\text { no planejamento do ciclo } \\
\text { básico; } \\
\text { - treinamento dos professores } \\
\text { para aplicação dos testes de } \\
\text { AVe estrabismo; } \\
\text { - Acompanhamento do progra- } \\
\text { ma, garantindo a realização } \\
\text { dos testes e esclarecendo } \\
\text { dúvidas dos aplicadores; } \\
\text { b) A realização do teste no inicio } \\
\text { do ano. }\end{array}$ & $\begin{array}{l}\text { a) Idem } \\
\text { b) Idem } \\
\text { c) O Departamento de Enferma- } \\
\text { gem da UFSCar prestaria } \\
\text { assessoria direta aos coorde- } \\
\text { nadores do ciclo básico na re- } \\
\text { alização das atividades acima. }\end{array}$ & $\begin{array}{l}\text { a) Idem } \\
\text { b) Idem } \\
\text { c) O Departamento de Enferma- } \\
\text { gem da UFSCar se responsabi- } \\
\text { lizaria pelo treinamento do coor- } \\
\text { denador geral do ciclo básico } \\
\text { da cidade de São Carlos, que } \\
\text { entäo treinaria todos os coor- } \\
\text { denadores do ciclo básico do } \\
\text { municipio que se responsabili- } \\
\text { zariam pelo acompanhamento } \\
\text { do programa, treinando e su- } \\
\text { pervisionando os professores } \\
\text { na execução do teste em suas } \\
\text { respectivas escolas. }\end{array}$ \\
\hline $\begin{array}{l}J \\
U \\
S \\
T \\
I \\
F \\
I \\
C \\
A \\
T \\
I \\
V \\
A \\
S\end{array}$ & $\begin{array}{l}\text { a) Por serem: } \\
\text { - Efetivos nas respectivas esco- } \\
\text { las, permitindo acompanha- } \\
\text { mento do programa e treina- } \\
\text { mento de novos professores; } \\
\text { - Responsáveis pelo planeja- } \\
\text { mento das atividades a serem } \\
\text { desenvolvidas pelo ciclo } \\
\text { básico, podendo incluir entre } \\
\text { elas o programa de AVnestas. } \\
\text { b) Nessa época: } \\
\text { - Os professores ainda năo } \\
\text { estāo sobrecarregados; } \\
\text { - O teste se torna mais eficaz, } \\
\text { pois detectado o problema } \\
\text { precocemente o aluno será } \\
\text { encaminhado, evitando as- } \\
\text { sim, comprometimento da } \\
\text { aprendizagem. }\end{array}$ & $\begin{array}{l}\text { c) Participação do Departamento } \\
\text { de Entermagem: } \\
\text { - Estímulo para execução do } \\
\text { teste; } \\
\text { - Maior garantia de fidedigni- } \\
\text { dade na aplicação do teste, } \\
\text { uma vez que o treinamento } \\
\text { seria mais intenso, corrigin- } \\
\text { do-se hábitos erróneos } \theta \\
\text { possíveis dúvidas. }\end{array}$ & $\begin{array}{l}\text { c) Participação do Departamento } \\
\text { de Enfermagem: } \\
\text { - Facilidade para o Departa- } \\
\text { mento de Enfermagem uma } \\
\text { vez que terá que treinar ape- } \\
\text { nas uma pessoa; } \\
\text { - Segurança na abrangência de } \\
\text { todas as escolas, pois a coor- } \\
\text { denadoria geral mantém con- } \\
\text { tato com todos os coordena- } \\
\text { dores da cidade de São } \\
\text { Carlos. }\end{array}$ \\
\hline
\end{tabular}

UFSCar - Universidade Federal de São Carlos.

O estrabismo foi encontrado em $2,2 \%$ das crianças testadas no presente estudo, o que é semelhante ao encontrado por Costa ${ }^{2}$, que obteve um percentual de 3,4\%. Apesar do estrabismo ter pre- valência baixa do ponto de vista absoluto (variando de 2 e $4 \%$ nas diferentes partes do mundo ${ }^{19}$ ), esses níveis são muito altos em termos sanitários. Sprissa e col. ${ }^{13}$ afirmam "que os desvios 
constantes são sempre anormais e o encaminhamento não deve ser retardado. Também o estrabismo constante pode ser sinal acompanhante de uma lesão ocular, às vezes grave como é o caso do retinoblastoma". As correçð̃es cirúrgicas de problemas tipo estrabismo a partir dos 7-8 anos de idade têm, na maior parte das vezes, apenas finalidade estética, devido a ambliopia dos anos precedentes levar a uma atrofia do nervo óptico. Portanto, a detecção de problemas oftalmológicos deve ser feita prioritariamente em berçários, creches e préescolas de forma geral ${ }^{12}$.

Não foi possivel verificar a relação entre queixas como ardor nos olhos e dor de cabeça, entre outros, com AV diminuída, devido as observaçðes não terem sido feitas de forma sistemática pelos professores. Gasperin e col. ${ }^{5}$ não encontraram relação entre tais queixas e AV diminuída.

A aplicação do teste de AV por professores é indicada por vários autores $4,5,6,12,15,16,18$. Especialistas reforçam a importância desses programas na escola como forma de prevenção e correção de desvios da normalidade, e destacam o papel fundamental do professor ${ }^{3,6,12}$. A Organização Mundial da Saúde reconhece que a prevenção à cegueira é uma atividade multidisciplinar e que deve conter ações nos três níveis de assistência à saúde.

A fidedignidade do teste de AV depende, em grande parte, do adequado treinamento das pessoas que vão aplicá-lo ${ }^{12,16}$. Nesse sentido, a proposta $B$, apresentada por professores na segunda fase do presente trabalho, faz referência à prestação de assessoria pela enfermeira ao coordenador do ciclo básico, cuja participação é fundamental para a institucionalização do programa. A enfermeira terá função de supervisão, assessoria e educação em saúde com a participação em programas a serem desenvolvidos ${ }^{9,10,18}$.

A proposta $C$ apresentada tem como incoveniente a centralização do conhecimento em uma pessoa, além de no decorrer da transmissão do mesmo haver o risco de este se diluir na passagem pelos diferentes níveis hierárquicos, comprometendo, assim, o resultado da aplicação do teste.

As propostas apresentadas (A, B e C) não são mutuamente exclusivas, ao contrário, elas se completam. Aos professores caberia a aplicação dos testes, aos coordenadores a definição, junto com os professores, das atividades a serem desenvolvidas como parte do programa em cada escola e ao coordenador geral do ciclo básico caberia a coordenação dessas atividades em nível municipal. Ao técnico, no caso a enfermeira, caberia a assessoria a esses três níveis, e especificamente em relação ao treinamento, caberia à enfermeira a capacitação para aplicação do teste por todas as pessoas nos diferentes níveis hierárquicos.

\section{Agradecimento}

À R. A. de Figueiredo, B. M. G. Martins e A.M.G. Sperandio pelas sugestōes e colaboração.

FIGUEIREDO, R. M. de et al. [A proposal for a procedure for the detection of ophthalmic disturbances in school children]. Rev. Saúde Pública, 27: 204-9, 1993. The proposal for a visual acuity test (A. V.) arose from a survey conducted among School Children of the " $\mathrm{Ci}$ clo Básico (C.B.)" i. é., the first two years of Elementary School in S. Carlos, S. Paulo State, Brazil. Nine schools participated in this study. The teachers were properly trained to apply the A. V. and squinting tests according to a standardized procedure. Of 2,025 children tested, $88.1 \%$ showed levels of $\mathrm{A}$. V. higher than 0.8 , and a squinting prevalence of $2.17 \%$. When the application of the test was over, each school presented its proposals for the systematical application of such tests. These proposals had a common point: the test should be applied by the teachers themselves under the supervision of the co-ordinators of the $\mathrm{C}$. B. The school nurse would be responsible for giving overall assistance to all the activities of the program at all levels.

Keywords: Vision tests, utilization. Mass screening, standards. School healt. Vision disorders, prevention.

\section{Referênclas Bibllográficas}

1. BEIGUELMAN, B. Curso prático de bioestatistica. São Paulo, Sociedade Brasileira de Genética, 1991.

2. COSTA, I. S. Educação em saúde escolar: análise de uma experiência. Rev. Bras. Enf., 27: 98-111, 1974.

3. FOCESI, E. Educaçāo em saúde na escola: o papel do professor. Rev. Bras. Saúde Esc., 1: 4-8, 1990.

4. GALOTTI, O. et al. Açōes que podem ser desenvolvidas visando à promoção da visão. In: Simpósio sobre Problemática da Cegueira, 3, São Paulo, 1976. Anais. São Paulo, p. 14-18.

5. GASPERIN, P. Jr. et al. Acuidade visual e visão cromática em escolares de primeiro grau em Curitiba. In: Prêmio Samuel Pessoa 1983. Curitiba, DANC-Departamento Científico Cultura//UFPR, 1985. p. 104-123.

6. JOSÉ, N. K. \& TEMPORINI, E. R. Ávaliação dos critérios de triagem visual de escolares de primeira série do primeiro grau. Rev. Saúde Pública, 14: 205-14, 1980.

7. JOSE, N. K. et al. Causas de deficiência visual em crianças. Bol. Of. Sanit. Panam., 97: 405-13, 1984.

8. KDNTZEL, K. C., ed. Advanced concepts in clinical nursing. Pensilvania, Lippicott Company, 1977.

9. NASCIMENTO, C. S. G. et al. A saúde da criança, riqueza do futuro: uma proposta de assistência de enfermagem em saúde escolar. Pediat. Mod., 20: 488-92, 1985.

10. RODRIGUES, M.A. Enfermeira em saúde escolar. Rev. Paul. Enf., 3: 50-3, 1983.

11. SECRETARIA DE EDUCAÇÃO. Departamento de Assistência ao Escolar. Oftalmologia sanitária escolar: as. pectos educativos. São Paulo, 1981.

12. SPERANDIO, A. M. G. Capacitação de professores e pajens para deteç̧ão precoce de problemas visuais - relato de uma experiência. Rev. Bras. Saúde Esc. 1: 22-5, 1990. 
13. SPRISSA, A. S. et al. Problemas oftalmológicas mais freqüentes de interesse pediátrico. J. Pediat., 61: 206$10,1986$.

14. TEMPORINI, E. R. Ação preventiva em problemas visuais de escolares. Rev. Saúde Pública, 18: 259-62, 1984.

15. TEMPORINI, E. R. Prevenção de problemas visuais de escolares: conduta de professores do sistema de ensino do estado de São Paulo, Brasil. Rev. Bras. Saúde Esc., 1: 68-72, 1990.

16. TEMPORINI, E. R. Programas de prevençăo da cegueira:participação da escola. Rev. Bras. Saúde Esc., 2: 41-3, 1992.
17. VAUGHAN, D. \& ASBURY, T. Oftalmologia geral. São Paulo, Atheneu, 1977.

18. VILLAR, M. C. et al. O que a enfermeira da Secretaria de Saúde vem fazendo pelo escolar. Rev. Bras. Enf., 37: 56-64, 1984.

19. WASSENBERG, C. Distúrbios visuais comuns em crianças. Nursing Clin. North Am., 16: 477-84, 1981.

Recebido para publicação em 27.4.1992

Reapresentado em 5.4.1993

Aprovado para publicaçäo em 31.5 .1993 